\title{
Dimensión Ética de la Educación y un enfoque para la Pedagogia
}

Nancy Chacón Arteaga ${ }^{1}$

\section{Resumen}

El trabajo aborda los aspectos conceptuales y metodológicos del enfoque ético, axiológico y humanista, elaborado por la autora en un trabajo sostenido de investigaciones en el campo de la Ética Aplicada a la Educación, en la Cétedra de Ética Aplicada a la Educación de su Universidad Pedagógica "Enrique José Varona", de la Habana, Cuba, llamando la atención sobre tan sensible aspecto de la espiritualidad humana y de la regulación, orientación y valoración de su comportamiento, como objeto de quienes contribuimos a la educación de los estudiantes, hijos y nietos.

Palabras claves: Ética, Educación moral y en valores.

\section{Summaries}

The work approaches the conceptual aspects and |metodológicos $\mid$ of the ethical, axiological focussing and humanist, elaborate by the author in a sustained work of investigations in the field of the ethics applied to the education, by attracting attention on so sensitive aspect of the human spirituality and of the regulation, orientation and valuation of your conduct, as object of whom contributes to the education of the students, children and grandchilds.

Code words: Ethics, moral education and in values.

\section{Una introducción necesaria: Por qué hablar de ética en estos tiempos de cambios de paradigmas?}

Ha sido un sueño de los grandes próceres de nuestra América, la necesaria integración a partir de los puntos comunes en que se gestara la identidad cultural de nuestros pueblos, por ende la necesidad de una integración en lo ideológico para tomar causa común en la lucha por la

\footnotetext{
${ }^{1}$ Profesora Titular, Dra. en Ciencias. Dra. Ciencias Filosóficas. Directora Centro de Ética Aplicada a la Educación Moral, en Valores y Ciudadana de la Universidad de Ciencias Pedagógicas "Enrique José Varona" de la Habana. Profesora Honoraria Universidad Autónoma de Santo Domingo UASD. Premio Nacional de Ciencias Sociales 2015.
} Interfaces da Educ., Paranaíba, v.9, n.27, p. 8-25, 2018 
independencia y soberanía, no solo del colonialismo europeo, sino del peligro en asecho y vía de hecho del imperialismo norteamericano.

Bolivar, el libertador, consciente de esta realidad se dio a la tarea de fundar esa gran patria latinoameriacana. Martí, continuador de las lecciones del padre fundador, no solo miró hacia las dolencias internas de la patria cubana adolorida aún bajo el yugo colonial español, sino que fue más lejos al considerar que "Cuba y Puerto Rico son de un pájaro sus dos alas", que "Del Río Bravo hasta la Patagonia una sola América", y que "Patria es Humanidad".

En su carta testamento escrita el 18 de mayo de 1895, a su amigo Manuel Mercado, desde Dos Ríos, Cuba, este le confiesa lo que en silencio había tenido que ser para "impedir a tiempo con la independencia de Cuba que se extiendan por las Antillas los Estados Unido y caigan, con esa fuerza más, sobre nuestras tierras de América". ${ }^{2}$

Es interesante reflexionar acerca de cómo estos estrategas de la independencia americana, a su vez, fueron capaces de comprender cómo el arma más poderosa para la unidad de voluntades y de esfuerzos personales para la lucha está en la sensibilidad humana cuya fibra interior está asociada al sentido de la justicia social, de la equidad, está en la dignidad que llevan consigo los hombres y mujeres con decoro, en la conciencia de los deberes de su época, en la solidaridad humana y entre los pueblos. En la base de esta toma de conciencia identitaria está la cultura, la educación, la moral y los valores.

Fiel exponente en la continuidad del ideario revolucionario, Bolívar le dice a su maestro Simón Rodríguez, "Usted formó mi corazón para la libertad, lo grande, lo hermoso" ${ }^{3}$. Bolívar preconiza la unidad de los dos polos necesarios los de "moral y luces", talento con moral, para la emancipación y el trabajo libertador.

Martí, al hablarnos de la Educación Popular, nos dice que "El pueblo más feliz es el que tenga mejor educados a sus hijos, en la instrucción del

\footnotetext{
2 Martí Pérez, José. Obras Completas T. 4 Edit. Ciencias Sociales, la Habana 1975, pág. 167

${ }^{3}$ Intervención de Hugo Chávez Frias el 17 noviembre 2003, sobre Educación Ciudadana en Venezuela
} 
pensamiento, y en la dirección de los sentimientos. Un pueblo instruido ama el trabajo y sabe sacar provecho de él. Un pueblo virtuoso vivirá más feliz y más rico que otro lleno de vicios, y se defenderá mejor de todo ataque." 4

En ambos la virtud está en la integración de los conocimientos y los nobles sentimientos, para saber correlacionar los fines que nos proponemos y los medios que empleamos para obtenerlos, son necesarios los conocimientos, el talento, así como los ideales humanistas, de justicia, independencia, libertad, dignidad, unidad y solidaridad, que llenan el pensamiento y las acciones emancipadoras de los pueblos y las personas.

Hoy en el mundo de globalización neoliberal, el problema de la crisis y degradación de los valores morales, es también resultado a la vez que causa de los problemas globales que afectan tanto a los seres humanos, como al medioambiente. En ello la agudización de la contradicción entre la ética del ser y la ética del tener y del todo vale, es una amenaza real de la absorción de los valores identitarios y culturales de los pueblos del tercer mundo, por las posiciones hegemónicas de una "super cultura y valores globales", que los países del primer mundo tratan de imponer a toda costa y a cualquier precio.

Ya desde mediados del pasado Siglo XX, el relevante científico alemán A. Einstein, hace un alerta premonitoria en su mensaje al 43 Congreso de la Sociedad Italiana para el progreso de las ciencias en 1950, conocido históricamente con el título de "La responsabilidad moral del científico", en la que expresó:

"Si los hombres de ciencia pudieran encontrar hoy día, el tiempo y el valor necesarios para considerar honesta y objetivamente su situación y las tareas que tienen por delante y si actuaran en consecuencia, acrecentarian considerablemente las posibilidades de dar con una solución sensata y satisfactoria a la peligrosa situación internacional presente."

Los valores humanos, son universales siempre y cuando respondan a los intereses genéricos de los seres humanos, cuando estos intereses se

\footnotetext{
${ }^{4}$ Martí Pérez, José. Obras Completas T. 19 Edit. Ciencias Sociales, la Habana 1975, pág. $375-376$
} 
quiebran y dividen a partir de lo material, los valores expresarán significados acerca de lo bueno y malo según los intereses y aspiraciones a que responden, sobre todo, si estos son de carácter económicos y políticos. Por lo tanto, lo más importante para la educación y el maestro (a), es defender la formación de valores humanos universales en correspondencia con la memoria histórica nacional y universal, las mejores tradiciones culturales, patrióticas y ciudadanas, que nos caracterizan como nación.

Rescatar esos valores donde se encuentren deprimidos, formarlos, desarrollarlos, promoverlos, entre otras acciones educativas, es la responsabilidad social que tiene hoy la escuela en cualquier parte del mundo; para asumir este reto, los educadores deben estar preparados y elevar su profesionalidad pedagógica, transformar su desempeño profesional y sus modos de actuación para cubrir estas expectativas de la pertinencia social y calidad educativa de nuestra gestión, en ello hay mucho que hacer y un camino largo por donde andar. En ese camino de cambios de paradigmas hacia la necesaria integración de lo común identitario en la diversidad multicultural de los pueblos latinoamericanos y caribeños, se inscribe la contribución del fomento de la Educación Ética y en valores, con una esencia emancipadora, libertaria, y endógena del desarrollo de las potencialidades humanas para la prosperidad y sostenibilidad del crecimiento armónico de nuestros países.

En la realidad de la Revolución cubana, conscientes de los peligros que nos amenazan, hemos aprendido las lecciones del pensamiento revolucionario cubano, desde nuestros padres y madres fundadores hasta la actualidad, quienes nos dicen que el conocimiento y la moral, son atributos esenciales de los seres humanos para la consecución de los fines, teniendo muy en cuenta los medios que se emplean para alcanzarlos. A continuación dos fragmentos del pensamiento martiano ilustran la presencia de importantes principios de la pedagogía de todas las épocas:

- Principio de la vinculación de la instrucción y la educación teniendo en la base de su interrelación a la moral.

"Instrucción no es lo mismo que educación: aquella se refiere al pensamiento, y ésta principalmente a los sentimientos. Sin embargo, no hay Interfaces da Educ., Paranaíba, v.9, n.27, p. 8-25, 2018 
buena educación sin instrucción. Las cualidades morales suben de precio cuando están realzadas por las cualidades inteligentes"( José Martí T. 19 O. C. pág. 375)

- Principio del vínculo dialéctico entre la educación y la época, cambiante e histórico concreta.

- Principio del carácter activo de la educación en la relación de la aprehensión del conocimiento y la vida práctica, cuyas necesidades son el motor del desarrollo de las fuerzas productivas, la ciencia y la tecnología.

"Al mundo nuevo corresponde la Universidad nueva.

A nuevas ciencias que todo lo invaden, reforman y minan nuevas cátedras.

Es criminal el divorcio entre la educación que se recibe en una época, y la época. Educar es depositar en cada hombre toda la obra humana que le ha antecedido: es hacer a cada hombre resumen del mundo viviente, hasta el día en que vive: es ponerlo a nivel de su tiempo, para que flote sobre él, y no dejarlo debajo de su tiempo, con lo que no podrá salir a flote; es prepararlo para la vida" (José Martí 1883 T. 8 pág. 281)

La educación como expresión genuina de la cultura, es el cimiento de lo que se espera de las nuevas generaciones para hacer próspera a la patria y a la humanidad. La Ciencia y sus resultados en las diferentes ramas y esferas de la vida, guardan una relación estrecha con la educación y sus propósitos que es la educación integral de las personas aportando la formación de los recursos humanos que se insertan como obreros, técnicos, profesionales, artistas o trabajadores de los servicios; por ello la Educación es el alma de las naciones y sus pueblos.

\section{Cultura ética, educación en valores y un enfoque}

“... hasta hoy toda moral ha sido, en última instancia, producto de las condiciones económicas de la sociedad en el periodo correspondiente. Y como hasta el día la sociedad se ha agitado entre antagonismos de clase, la moral ha sido siempre una moral de clase; o justificaba la 
dominación y los intereses de la clase dominante, o representaba, cuando la clase oprimida se hacía lo bastante poderosa, la rebelión contra esta dominación asi como los intereses del futuro de los oprimidos."

El desarrollo científico y tecnológico que alimenta el proceso de globalización del capitalismo neoliberal, trae consigo un enfoque tecnocrático y pragmático de la educación y de la formación de los profesionales en particular, que sobredimensiona los conceptos de sociedad del conocimiento, desempeño, competencias, competitividad, éxito, calidad, entre otros, los que tienen un cierto carácter elitista y en su connotación ideológica excluyen el contenido ético, axiológico, humanista y medioambiental, en la visión integradora de la formación de la profesionalidad de los mismos, estos criterios éticos solo se aplican como grupo corporativo, compañia, firmas, agencias, entre otros, que pueden cambiar y de hecho son diferentes entre sí, estos van a responder a los intereses específicos de cada grupo, por lo que no constituye un objetivo a lograr en la formación moral de la persona como profesional para la sociedad en que vive, este aspecto concierne más bien a lo personal, el marco familiar y religioso.

La cultura ética abarca el conocimiento sobre la esencia, el lugar y papel de la moral y los valores en la vida social en la regulación del comportamiento humano, lo que se expresa en la educación moral que manifiesta la persona y las cualidades morales que la distinguen; la misma tiene como base las normas elementales de la convivencia en la comunicación, las relaciones humanas y con el medio ambiente.

La aplicación intencionada de los conocimientos éticos, como parte de la visión del mundo en que vivimos, de las representaciones del bien y del mal, de la moral en que nos desenvolvemos en los diferentes ámbitos de nuestras vidas personal y social, nos lleva a tener en cuenta el factor moral en todo lo que hacemos, transformándose en un enfoque como un valioso instrumento en la regulación autodeterminada de la conducta y de la dirección de los procesos sociales; este es el núcleo de la cultura ética, la que

\footnotetext{
${ }^{5}$ Engels Federico. Anti Duhring. Pueblo y Educación, Habana 1975. Página 116 Interfaces da Educ., Paranaíba, v.9, n.27, p. 8-25, 2018
} 
no solo se adquiere por el conocimiento académico de esta disciplina, sino sobre todo, por la práctica de la moralidad y sus influencias en la trasmisión del código moral vigente en la sociedad (principios, normas, costumbres, tradiciones), que permiten orientarnos, educarnos, discernir entre el bien y el mal y tener un sentido común, como parte del sentido de la vida de las personas.

En los tiempos que corren, es muy importante apropiarnos del enfoque ético, axiológico y humanista (EAH), que nos aporta la Ética sobre la moral y su papel en la sociedad y en el mundo interno de las personas, donde los valores morales como componentes de la conciencia moral, se transforman en brújulas orientadoras de las escalas valorativas y de las preferencias jerárquicas de las actitudes que se asumen ante al mundo en que vivimos por medio de la autorregulación consciente de la conducta, siempre en franca contraposición entre el hacer u obrar bien o su contrario, actuar sin tener en cuenta los intereses de los demás o transgrediendo las normas morales aceptadas socialmente.

El enfoque ético - axiológico de orientación humanista, considera las potencialidades del ser humano como un sujeto activo para autoeducarse en la sociedad en que vive, según las normas, costumbres y tradiciones vigentes, que se trasmiten de generación en generación, en primer orden en el seno familiar, así también para crecerse ante las circunstancias más adversas o de resistencia.

La moral y los valores, argumentan o justifican nuestros actos de conducta, nos hacen sentir la satisfacción de ser personas decentes cuando sabemos comportarnos, relacionarnos y comunicarnos con los demás practicando las normas de cortesía y convivencia. Cuando somos conscientes de haber cometido un error o de haber violado una norma en perjuicio de otras personas, la autoconciencia moral genera un sentimiento de culpa y de vergüenza, en ello tiene mucho que ver la importancia que la autoestima de cada quien le da a la fuerza de la opinión pública, es decir, qué tan bien valorados o no somos en la aceptación o el rechazo que provocan las cualidades, las actitudes y el comportamiento que nos caracterizan ante los demás, es el sello de la imagen pública. 
A su vez, la moral también permite sentir la insatisfacción crítica con la realidad en que vivimos y modelar anticipadamente el presente en su visión futura para ser mejorada a mediano o largo plazo en la propia actividad que realizamos, lo cual se modela en las representaciones del deber ser y en los ideales morales, de acuerdo a los intereses de los individuos, grupos o clases sociales, sus necesidades, motivaciones, fines y anhelos; es por ello que hoy más que nunca debemos seguir hablando de ética, moral y valores humanos, como elementos esenciales de la educación, de la cultura y de la ideología de la Revolución cubana.

\section{Sobre la Dimensión Ética de la Educación}

De la influencia del ilustrado pensamiento greco - romano de la antigüedad, nos llega el legado de Aristóteles, reconocido como el "Padre de la Ética" por haber sido el primero en dedicar un acápite de su enciclopédico conocimiento filosófico a la Ética, en un singular libro que dedicó a su hijo y por ello denominó "Moral a Nicómaco"; en este tratado reflexionó sobre qué debía hacer el hombre y de qué debía de abstenerse para llegar a ser una persona virtuosa, en ello insistió en que las virtudes se forjan asociadas al carácter del ser humano por lo que debian formarse desde las edades tempranas, y apuntaba que esto se logra en el "hábito", es decir en el actuar de cada día.

Aún las distancias temporales, de épocas y conceptuales que nos separan de estas ideas aristotélicas, las mismas guardan una gran vigencia, pues en ellas hay mucho de razón y de sentido común cuando de la educación de la moral en la práctica de las virtudes se trata, lo que apunta a que desde tiempos remotos este ha sido un problema que la humanidad ha enfrentado de diferentes maneras pero siempre con una regularidad: la educación moral y de valores está en la base de la sucesión de generaciones en cuyo núcleo central está la relación recíproca que en este sentido se da entre padres e hijos, de los mayores hacia los menores, entre educadores y educandos. 
Esto nos lleva a la idea de que la educación como proceso formativo o como institución social encierra en su contenido una dimensión ética que está presente desde la propia intención o propósito humano de influir positivamente en otras personas para enseñarles el Bien, esto es, aprender a ser seres humanos y a vivir armónicamente en una familia dentro de su contexto social, aprendizajes que en general encierran conocimientos, habilidades, afectos sentimientos, cualidades y formas de comportamientos.

En Cuba, El Sistema Nacional de Educación vive momentos de cambios trascendentales, marcado por un proceso integral de reordenamiento económico e institucional en el país a la luz de la actualización del modelo económico, así como por un Perfeccionamiento que conduzca a un Modelo Educativo que propicie un salto cualitativo en la formación de los niños, jóvenes y adultos en todos los subsistemas y centros educacionales, a partir del tipo de escuela que se necesita en estos tiempos y de maestro, donde la sociedad no solo espere una respuesta ante el encargo que demanda de la educación, sino que sea partícipe del logro de tan caro objetivo educativo, que cubra las expectativas y niveles de insatisfacciones sociales, así como las realizaciones en lo personal de sus protagonistas: un estudiante con educación y un educador con profesionalidad.

Para lograr el perfeccionamiento de la Educación cubana en el contexto actual es necesario, entre otros aspectos, potenciar y fortalecer la dimensión ética de la educación ${ }^{6}$, la que expresa el significado del contenido ético, axiológico y humanista del proceso educativo, manifiestado en el lugar y papel que tienen la moral, los valores morales y el humanismo en todo el proceso formativo de los seres humanos, presente tanto en los fundamentos teórico, normativo y práctico de la profesionalidad del docente

${ }^{6}$ El término de Dimensión ética de la educación, aparece tratado por la autora en el folleto elaborado para la formación experimental de los Profesores Generales Integrales, publicado por Pueblo y Educación en el 2000, en el mismo se abordan las ideas esenciales del enfoque ético, axiológico y humanista aplicado a la educación en sus conceptos claves. Posteriormente en el 2002 se hace una edición de un texto más amplio donde la autora incorpora artículos de los doctorantes del proyecto de Ética y profesionalidad que asesoraba y que amplían la concepción del enfoque ético, axiológico y humanista; finalmente en el 2006 se publica una tercera edición del texto con formato de libro, donde se renueva la estructura de los capítulos y los contenidos del enfoque ético, axiológico y humanista y sus aplicaciones en los aportes de los coautores en sus tesis de doctorado.

Interfaces da Educ., Paranaíba, v.9, n.27, p. 8-25, 2018 
en su desempeño como educador, así como en la pedagogía que sustenta la educación moral, en valores y ciudadana de sus estudiantes como base de los objetivos formativos o perfil del egreso en el grado o carrera de que se trate.

Esta dimensión ética de la educación, focaliza la horizontalidad y transversalidad que tienen, la moral, los valores morales y el humanismo, en este proceso, que no se reducen solo al ángulo normativo de la "Ética pedagógica", con el que por lo general muchos asocian la aplicación de la Ética al campo de la educación y de la pedagogía, posiciones ya superadas en esta concepción de la Ética Aplicada a la Educación, lo que se evidencia en:

- Los fundamentos ético - morales y axiológicos de las políticas educacionales.

- El enfoque ético, axiológico y humanista EAH, como fundamento de la Pedagogía tanto en la teoría y la práctica de una visión más integral e integradora de la educación, vinculada con los enfoques sociológicos, históricos, antropológicos, psicológicos e incluso de la tecnología educativa o aplicada a la educación, entre otros.

- La teoría y práctica de la educación moral y ciudadana, metodologías, medios, estrategias.

- La presencia de la moral y los valores en la esfera humana de las múltiples relaciones, de la comunicación y el trato interpersonales entre todos los implicados en el proceso educativo.

- Como parte del clima moral del escenario de la escuela y del contexto socioeconómico de la familia y de la comunidad.

- Formando parte del sistema de influencias de múltiples factores en la esfera moral de las personas en la sociedad, incluyendo las redes sociales, los grupos informales, los medios de comunicación entre otros.

- Presencia en los procesos sustantivos de la escuela o la Universidad.

- Presencia en el currículo de la formación permanente (inicial y contínua). 
- En el empleo de las TIC como medios educativos y herramientas para la vida, en el respeto al otro y al medio ambiente.

- Como exigencias a la profesionalidad del docente en sus saberes y competencias en sus desempeños y modos de actuación en su trabajo con apego a los principios, normas y valores de la profesión.

- En el comportamiento moral y ciudadano del estudiante.

- En las didácticas de las disciplinas y los contenidos de la enseñanza, métodos humanizados, democráticos - participativos, autorreguladores.

El desarrollo de la ciencia y la tecnología actual en un mundo cada vez más objetivamente "global" - evidente a la vista de todos - hace que la Educación y la Pedagogía como ciencia rectora del proceso educativo, afronten múltiples retos, uno de ellos es la forma fragmentaria con que aún se concibe tanto en la teoría como en la práctica pedagógica este complejo proceso, de carácter multidisciplinario, interdisciplinar y multidimensional en el que intervienen muchas variables $\mathrm{y}$ factores tanto internos como externos comprometidos -al menos en lo formal y normado- con el propósito esencial de la educación integral de las nuevas generaciones.

El tratamiento intencionado y explícito de la Dimensión ética de la educación como un contenido nuclear de la Pedagogía de todos los tiempos, puede contribuir a principios reguladores internos del proceso educativo tales como: la coherencia, la direccionalidad, la intencionalidad y la sistematicidad, los que requieren hoy más que nunca la convergencia de la palabra y la acción como base del prestigio moral del maestro en la integración del complejo proceso de instruir y educar a nuestros niños y jóvenes, que como dijera José Martí en su concepto de Educar, los prepare para la vida. Lo anterior nos permite integrar las ideas que explican qué entender esta idea y su complejidad: La Dimensión ética de la Educación, es un concepto cuyo contenido expresa el lugar y el papel que tienen las ideas y concepciones éticas de la cultura de la época, el sistema de la moral y los valores morales vigentes, en el proceso educativo, como fundamento ético - axiológico y humanista del fin y esencia de la educación, presente en la dinámica entre el Ser moral o la moralidad practicada en la Interfaces da Educ., Paranaíba, v.9, n.27, p. 8-25, 2018 
convivencia social y las representaciones del Deber Ser y el Ideal moral de la profesión, a que se aspira en los modelos educativos y modelo del egresado de cada carrera y le imprimen una proyección valorativa y de regulación moral hacia el desarrollo humano como idea de una tendencia del progreso humanista en el proceso formativo.

A su vez, en la dimensión ética de la educación, se revelan las interinfluencias y relaciones morales que se producen entre las personas, agencias y actores educativos en los diferentes escenarios y contextos de la educación, con sus particularidades de manifestaciones en las cualidades morales de dichos sujetos (docentes, estudiantes, familia, comunidad), en la subjetividad o mundo interior, en la educabilidad y sensibilidad humana, en la actitud y las relaciones ante lo que nos rodea, la comunicación, en la resolución de los conflictos morales y en las normas presentes en la elección del acto moral, concretada en los modos de actuación y los comportamientos asumidos, los que dicen del grado de educación moral de la persona y su cultura ética.

\section{Algo más sobre la educación moral y en valores de nuestros niños}

Toda sociedad tiene en sus niños y jóvenes su tesoro más preciado, pues somos conscientes de que ellos representan la continuidad generacional de quienes hoy somos sus padres, abuelos e incluso maestros y por ende en ellos - como dijo José Martí en su revista "La Edad de Oro"- está la "esperanza del Mundo".

En esa esperanza se cifran los anhelos de que al crecer ellos sean mejores que nosotros, más cultos y preparados para desempeñarse competentemente en su trabajo, a la vez que educados. La educación está en la base de este crecimiento humano, en particular de la moral y los valores morales, los que están en el centro a la calidad de la persona, empezando por sus sentimientos y cualidades que lo distinguen en sus relaciones, la forma de expresarse en la comunicación con su familia, los demás y el trato. 
No podemos olvidar que la moral es una importante esfera de la regulación y la autorregulación consciente de las actitudes y del comportamiento humano en la sociedad y que la autoconciencia moral de cada persona permite la valoración de todo lo que sucede tanto en lo personal como a otras personas, lo que acontece a nuestro alrededor o en el mundo. Esta valoración es la que proyectamos en nuestros puntos de vista y juicios valorativos, los que siempre tendrán el sello personal de nuestra mirada, subjetividad e intereses, aspectos inherentes a la esfera de la moral y donde los valores personales organizados en escalas jerárquicas juegan el papel de orientadores no solo de la valoración, sino unido a ello de la actitud y el comportamiento que asumimos ante los hechos o circunstancias de la vida cotidiana.

Es por ello que la educación moral y de los valores morales inherentes como brújulas orientadoras, están en la base de toda educación y es un aspecto integrador de los conocimientos y habilidades con su aplicación en la vida práctica, en el cómo obrar, qué comportamiento elegimos ante los conflictos o dilemas que se presentan en el día a día.

La familia tiene un papel singular en la educación moral de los niños desde antes de su nacimiento, esta, la familia, los acompaña día y noche en lo que es esa "escuela - hogar", las costumbres y las normas, los sentimientos y afectos expresados en el lenguaje, las formas de la comunicación y de relacionarse, son esenciales, en ello están los modelos que por excelencia primero imitan los niños y luego poco a poco interiorizan como suyos propios, en su moral y así será como se expresan y se comportan en la escuela, con sus compañeros, maestros, en la cuadra con sus vecinos o en los lugares públicos.

Obviamente estamos ante un problema nada sencillo pues se trata de los seres humanos y de su educación moral, proceso que transcurre en el contexto de la sociedad y en el que intervienen múltiples factores e influencias, en una época impactada por el desarrollo científico y tecnológico, donde hay quienes piensan que este tema no hay que tomarlo muy en cuenta y que es un asunto de la vida privada de cada persona. Nada más lejos de la verdadera importancia que tienen la ética, la moral y los Interfaces da Educ., Paranaíba, v.9, n.27, p. 8-25, 2018 
valores morales, tanto en la convivencia humana y con el medio ambiente, como en el acompañamiento al desarrollo científico - técnico, sus contracciones y conflictos.

Pero en este plano familiar del análisis podemos preguntarnos: cuáles son esas formas de encauzar esta educación o los métodos invisibles con que la familia educa a sus hijos en el hogar?

Dentro de estos cobra una gran significación el modo de organización de la vida de la familia y en particular de los niños sobre la base de sus derechos y deberes, unido a esto están los hábitos elementales de educación moral de la convivencia en el hogar ante cada actividad conjunta que hacemos todos los días, con el amor, la constante conversación dialógica, la persuasión explicativa y la comprensión que reclama cada uno de nuestros hijos y sus inquietudes. Un papel especial tiene el modelo o ejemplo que observan en la actuación de sus padres o tutores con apego a estas normas sutiles practicadas en un ambiente de afecto reciproco, lo que genera un proceso de admiración sentida e identificación con esos patrones en la actuación.

Esta educación en la propia práctica de las normas y valores en las costumbres y tradiciones, se van transformando en experiencias morales acumuladas como parte de sus vivencias personales y potencian la aprehensión de los conocimientos acerca de lo que es bueno y es malo, lo justo o injusto, lo honesto o deshonesto, entre otras representaciones y significaciones de los valores y antivalores del mundo moral en que viven, contribuyendo al crecimiento humano desde una educación con una autorregulación moral basada en el respeto a la dignidad y el significado de las normas morales básicas de las relaciones y la convivencia. Es importante que haya confianza en la autonomía, independencia o autodeterminación que va conquistando en su desarrollo, pero siempre con el debido control y la ayuda de sus seres queridos, en relación estrecha con los maestros y la escuela.

En este complejo y no menos contradictorio proceso educativo, no puede faltar la idea del protagonismo e independencia responsable, que nuestros hijos deben conquistar en cada momento, asumiendo sus deberes Interfaces da Educ., Paranaíba, v.9, n.27, p. 8-25, 2018 
escolares y de las actividades diarias de colaboración en la casa y con sus familiares, sin paternalismos o sobre protección como estilos negativos que pueden hacerlos dependientes, perezosos, irresponsables, desconsiderados y lo que es peor seres egoístas.

Hoy es muy dado que los padres consideren que la educación de sus hijos y su felicidad está asociado a lo que materialmente le pueden dar, con independencia de lo que ellos hacen en el cumplimiento de sus deberes, descuidando el $\mathrm{ABC}$ de lo que nos ocupa que es la educación moral, como parte de su espiritualidad y de sus sentimientos, esto es lo más valioso del ser humano, esa es la "semilla escondida" que todos debemos hacer florecer.

Al referirse a la educación de nuestros hijos tengamos siempre presente la máxima moral y humanista que encierra la dedicatoria que José Martí escribiera a su hijo José Francisco en su dedicatoria de los versos del "Ismaelillo" cuando expresó: "Hijo: Espantado de todo, me refugio en tí. Tengo fe en el mejoramiento humano, en la vida futura, en la utilidad de la virtud, y en ti". ${ }^{7}$

\section{Una reflexión final}

Aún lo controversial y polémico que resultan estos temas educativos en la realidad del mundo de hoy y en los contextos diferentes, puede afirmarse que la Pedagogía ha avanzado en los fundamentos teóricos y metodológicos que aborda el complejo proceso de la Educación en valores, que va desde las tempranas edades, hasta los propios docentes en su formación permanente y su profesionalidad, sin embargo, aún se necesita escudriñar los aspectos multifactoriales e interdisciplinarios que se entrecruzan en la horizontalidad de este proceso y que tienen junto a los conocimientos y las habilidades para operar con ellos, a los valores morales y de las ciencias, los que por lo general son aglutinadores de la diversidad de tipos y sistemas de valores, escalas y preferencias jerárquicas, para asumir una orientación y una actitud valorativa ante el mundo en que se convive

\footnotetext{
7 Martí José, Ismaelillo, O.C. Editorial Ciencias Sociales. Habana 1975 T. 16. p. 15 
entre los seres humanos y con la naturaleza, en la que cada persona se inserta con un sentido propio de la vida, autoestima, autodeterminación y autorregulación responsable, como bases de la formación ciudadana a la que aspira todo país y sistema educacional, respecto a sus nuevas generaciones. En esa visión cada vez más integradora del proceso formativo, el enfoque ético, axiológico y humanista hace junto a otros, una nueva aproximación a su esencia.

\section{Bibliografía básica}

Buch Rita M. Los precursores en la Filosofia de la Educación cubana. Conferencia inaugural VIII Taller nacional martiano de ética "Ciencia, Conciencia y Valores”. Casa Cultural del Alba. La Habana 20/6/13.

Castro Ruz Fidel. Discurso Aula Magna de la Universidad de la Habana el $17 / 11 / 2005$.

Discurso en la graduación del IX Destacamento Manuel Ascunce Domenbech. Ciudad Libertad. La Habana, 7/7/1981.

Fragmentos de discursos publicados 1959 - 2006. Multimedia

Fidel: Ética y valores humanos. CESOFTE UCPEJV. La Habana 2007.

Colectivo de autores ICCP. Pedagogía. Editorial Pueblo y Educación. La Habana1998.

Chávez, J. y Báxter, E. La educación en valores y la relación con la evaluación de su calidad. Pueblo y Educación. La Habana 2008) Chacón Arteaga Nancy. Martí y Fidel en el código de ética del educador cubano. Pueblo y Educación. La Habana 2013.

y colectivo. Dimensión ética de la Educación cubana. Pueblo y Educación. La Habana 2006.

Moralidad histórica, valores y juventud. Editorial Acuario. La Habana 2000.

Formación de valores morales. Editorial Academia. La Habana 1999. y colectivo. Educación, Ciencia y Conciencia. Pueblo y Educación. La Habana 2008. 
y colectivo. Sistematización de resultados de proyectos de investigaciones de la cátedra de Ética Aplicada a la Educación. Universidad Pedagógica Enrique José Varona. La Habana 1998 - 2014.

Engels F. Anti Duhring. Pueblo y Educación 1975.

Hart Dávalos Armando. Ética, Politica y Cultura. Con la honda martiana. OPM. Habana 2009

Guevara de la Serna Ernesto. Che Guevara presente. Antología mínima.

Ocean Press. 2005

Martí Pérez José. Obras Completas. T. 8, 19 y 22. Ciencias Sociales. La Habana 1975.

Marx y Engels. Obras Escogidas en III Tomos. Moscú 1975

Programa Director de Educación en el sistema de valores de la Revolución cubana. CC PCC. La Habana, mayo 2012. 